\title{
Microarquitectura Urbana Inmótica. Propuesta de diseño útil, organizado y articulado, atendiendo la sustentabilidad y la inclusividad, para espacios intersticiales de la ciudad de Córdoba
}

Inmotics Urban Micro-architeture Design proposal useful, organized and articulated, paying

attention to sustainability and inclusion, to interstice spaces of the city of Córdoba

\author{
DRA. ARQ. Silvia Patricia Hernández \\ Faud, UNC. Argentina \\ arqpatriciahernandez@gmail.com
}

D ESP. ARQ. Gabriela Mengo

Faud, UNC. Argentina

\author{
> ESP. ARQ. María José Verón \\ Faud, UNC. Argentina
}

> ARQ. Luciana Lanzone

Faud, UNC. Argentina
> ARQ. María Figueroa

Faud, UNC. Argentina

> ARQ. Alejandra Rezk

Faud, UNC. Argentina

\begin{abstract}
The following paper continues with the evaluation of technologies used for the application of domotics in our country, Argentina. It was proposed to work in microarchitecture, applying it to proposals with useful design for specific urban spaces called residuals or interstitial. With this purpose an analysis was done with several existent examples of microarchitecture, systematizing the contributions of automation, regulation and computerized control that they may have, and assessing achievements of sustainability and user's comfort. It was started to propose urban social typologies, for the whole society, as in information centers, experimentation, vaccination center, etc. The work is in progress.
\end{abstract}

Keywords: Inmótic - Urban micro-architeture- Spacial Insterstice- Sustainability

\section{Introducción}

Con la presente investigación se continúa con la evaluación de las posibilidades, modos y tecnologías utilizadas para la aplicación de la domótica en nuestro país, Argentina. Se propuso investigar y desarrollar en este proyecto con los avances tecnológicos y de diseño que se están dando en el mundo y en Argentina en cuanto a micro arquitectura aplicándolo en propuestas concretas de diseño útil para determinados espacios urbanos llamados residuales o intersticiales. La propuesta será articulada y organizada de acuerdo a los planes y normas que regulan la ciudad de Córdoba. A estos efectos se realizó un análisis de ejemplos de micro-arquitectura existentes, sistematizando los aportes de automatización, regulación y control informatizado valorando el logro de sustentabilidad y el confort de sus usuarios, comprobando y relacionando la transformación y utilidad posible para esta ciudad.

En base a esta sistematización y luego de una evaluación de rendimientos se propondrán tipologías sociales urbanas, con prioridad para que sean utilizadas por toda la sociedad, como centros de información, de experimentación, de divulgación, vacunatorios, etc. Estas tipologías serán ejemplos de micro-arquitectura domótica, que contemplen la inclusión de automatizaciones y de la domótica tanto en sus equipos como en sus envolventes, conforme a los requerimientos funcionales, morfológicos, de sustentabilidad y técnicos propios, considerando las características socioeconómicas y constructivas del medio.

\section{Domótica / Inmótica}

La evolución histórica indica que en un principio los sistemas aplicados en las construcciones eran de control manual. En los Estados Unidos, tras la crisis petrolera de los ańos 70, con el objetivo principal de generar un ahorro en el consumo, surgen las primeras automatizaciones en edificios, (Romero Morales et al, 2006). Luego, de las primeras automatizaciones con el uso de los termostatos, se comenzaron a usar los sensores para los controles de humedad, caudal de aire, etc., en los sistemas de climatización, y para los controles de intrusos, en los sistemas de alarmas.

Se la llamó domótica, que proviene de domus: casa y tica: de domotique (del francés): robótica (Enciclopedia Larousse, 1988).

El término domótica tiene una génesis análoga a la del término informática, sustituyendo el prefijo que significa información por otro derivado de la palabra latina domus, que significa casa. También reciben un trato análogo en la bibliografía en lengua inglesa, en la que son más comunes otros términos como computing en lugar de informática o smart house y intelligent building en lugar de domótica. (Recuero, Caminos, 1999).

La domótica surge entonces con el objetivo principal de otorgar al usuario el máximo confort y seguridad con la mayor economía y eficiencia energética.

Cuando se orienta a edificios terciarios, no viviendas, estamos ante la presencia de la inmótica, que realiza la gestión de la energía incluyendo las automatizaciones de las actividades y el trabajo. (Morales, Serrano, Lozano, 2006). Esto incluye la ofimática como 
un subsistema. La ofimática es el conjunto de técnicas, aplicaciones y herramientas informáticas que se utilizan en funciones de oficina para optimizar, automatizar y mejorar los procedimientos o tareas relacionados. (Computer Cience and Communications Dictionary, 2001).

En este trabajo usaremos el término inmótica, que es la nominación específica para la domótica cuando la tipología no es vivienda y dejaremos el uso del término domótica para las generalidades.

\section{Espacios residuales, Instersticios espaciales}

Reconocemos en la compleja trama urbana de las ciudades espacios llamados residuales que en realidad son instersticios espaciales. Considerando entonces como espacio intersticial al espacio que media entre dos partes de distintos objetos o entre dos del mismo cuerpo.

En la tarea de aproximarse a nuevas lecturas operativas sobre el territorio, se trabaja a partir de investigar los procesos actuales que intervienen en la espacialidad intersticial en términos de reinterpretación como potencial de nuevas propuestas. Se trata de nuevas configuraciones que se trabajarán con la adaptación, o engrosamiento o completamiento de estos intersticios.

Se tomarán en cuenta a los habitantes que serán usuarios de todas las edades, y con capacidades espaciales, proyectando con inclusividad. En las ciudades a los niños se los considera habitualmente con los juegos infantiles, como si no pudieran hacer otros usos.

El mobiliario urbano es todo aquello que amuebla la calle, todo lo que, en el interior de una ciudad o de una aglomeración, se encuentra erigido en el borde de las vías, sobre las aceras, o sobre la misma vía pública.

El equipamiento urbano, algo más que el mobiliario urbano, ya que contiene, y quizás no está ubicado en la acera, es sostenible cuando se integra con el paisaje y a la vez genera energía limpia a través de paneles solares y/o eólica. Se trata de articular servicios para la vida urbana y para la naturaleza.

Los elementos urbanos identifican la ciudad y a través de ellos podemos conocer y reconocer las ciudades.

\section{Propuesta}

Nuestro objetivo fue realizar una propuesta de diseño de micro-arquitectura urbana, con desarrollo tipológico de servicio para la ciudad, con tecnología domótica, autosustentable, que sea inclusiva, para un espacio intersticial público urbano determinado de la ciudad de Córdoba, entendiendo su organización, y articulación con la ciudad, y desarrollada con tecnología local, considerando los avances tecnológicos y de diseño que se están dando en el mundo y en Argentina.

\section{Objetivos}

1. Elaborar un diagnóstico del estado actual del diseño y producción de micro-arquitectura domótica y sustentable, en tipología desarrolladas en espacios intersticiales, en el mundo y en argentina.

2. Elaborar categorías que permitan configurar los aspectos implicados en cuanto a diseńo, prestaciones, confort, economía, seguridad, diseño inclusivo y grado de tecnología aplicada para espacios transformados en útiles.

3. Evaluar los modos de funcionamiento de los equipos domóticos existentes en estas tipologías, en cuanto al modo en que se realiza la automatización, regulación y control de: energía, optimización de la función, redes informáticas, iluminación, climatización, ductilidad, y otras variables y su relación con las centrales de control.

4. Estudiar los comportamientos de los usuarios, la interacción propuesta, las preferencias y rendimientos.

5. Desarrollar tipologías de diseño y de este ejercicio generar pautas que den fundamento a las tendencias de diseño y producción en nuestro país cumpliendo los objetivos de esta investigación, de acuerdo a las demandas del medio y la innovación tecnológica.

6. Realizar un diagnóstico del resultado del comportamiento de los sistemas descriptos en -2 y su impacto en el medio socio- económico y ambiental.

Sitio

Se eligió un área de intervención que reuniera condiciones urbanas específicas. Nuestro proyecto se ubica en un espacio entre dos carriles de la misma calle, el Bv. Illia, Este corredor divide físicamente y topográficamente el centro de la ciudad con el barrio Nueva Córdoba, quedando el Mercado Sur al límite con una barrera en frente que divide a su vez el boulevard.

El Mercado Sur que hoy es un área protegida (zona especial según normativa 8057-08) desde el aspecto histórico - urbano patrimonial del centro de la ciudad con gran potencial turístico, cultural y comercial.

El área del mercado y sus alrededores es un área problemática porque se encuentra a 300 metros del centro histórico como un ente independiente o cerrado que no ofrece intercambios ni actividades culturales y sociales.

Se detecta falta de espacio público/verde en la zona.

Lo que nuestra propuesta intenta resolver es potencializar la zona a través de la incorporación de un objeto arquitectónico que proponga actividades y servicios para mejorar la calidad de vida de los ciudadanos generando un nuevo polo de referencia en el microcentro urbano proponiendo un mixtura de usos que defina espacio público/ de servicios. A su vez, este polo se puede clonar en otros sitios intersticiales de la ciudad buscando condiciones urbanas similares.

\section{Función}

\section{Espacio Público}

Según la Real Academia Española (RAE, 2014) básicamente, y a diferencia de la propiedad privada, el espacio público está libre de construcciones, a excepción de equipamientos colectivos (recreativos, cívicos, culturales de intercambio, referente simbólico etc.) y contiene además servicios públicos con destino a usos sociales o cívico comunitario inherentes a la vida urbana.

Desde la dimensión socio-cultural el espacio público asume el rol de soporte de relación, de encuentro, de construcción de la identidad 
ciudadana, de expresión comunitaria. El comportamiento de la gente y en este sentido la apropiación en sentido de pertenencia a un determinado espacio público, le confiere un uso que lo consolida como tal.

Por consiguiente la esencia del espacio público se sustenta en el dominio público, el uso social y colectivo y la multifuncionalidad, siendo asimismo sus características físicas, la accesibilidad y su calificación ligada proporcionalmente a la cantidad y calidad de las relaciones sociales

El espacio urbano tiende a nuevos procesos de concentración y complejización de actividades y usos.

Se define Confort Urbano como el conjunto de condiciones óptimas que deben coincidir simultáneamente en un espacio público para lograr su máximo aprovechamiento o disfrute para una actividad y un momento concreto.

El Confort en el espacio público urbano viene determinado por distintos factores: condicionantes térmicos, escala urbana, ocupación del espacio público, paisaje urbano, percepción de seguridad, condiciones acústicas, calidad del aire, ergonomía. Todos estos parámetros están interconectados. La alteración de uno de ellos repercute en la calidad de los demás, (Cabezas, 2014).

\section{Antecedentes}

Luego del análisis detectamos Las características de todo elemento de micro-arquitectura urbana son:

- $\quad$ Estética

- Solidez y duración

- Funcionalidad

- Idoneidad de instalación

- Facilidad de reparación

- Facilidad de mantenimiento.

- Respetar la ordenanza municipal

- Ofrecer al usuario ciudadano: Confort, Protección y separación, Información o comunicación, Reposo e higiene

- Interacción del usuario con los objetos y el espacio

- Interacción del objeto/espacio propuesto con la ciudad

\section{Antecedentes analizados}

Se estudiaron diversos ejemplos de microarquitectura. Presentaremos sólo dos

\section{Antecedente uno}

El MODULO, Abitare al 11, de Alberto Martínez, presenta una propuesta longitudinal, compuestas de módulos de estar sociales y módulos de servicio.

\section{Antecedente dos}

La propuesta de Kiosko ABITARE, da una tipología adaptable a muchas funciones. Aquí presentamos el módulo heladería desarrollado en acero galvanizado de $3 \mathrm{~mm}$, con revestimientos para aislaciones térmicas, con terminaciones lacadas. Los solados y otras envolventes en madera de Iroko. Proponen una opción de vidrio laminado $8+8$, con retroiluminación y soporte publicitario, como lo venimos proponiendo en nuestros diseńos de microarquitectura ya presentados. Propone automatizaciones en las persianas.

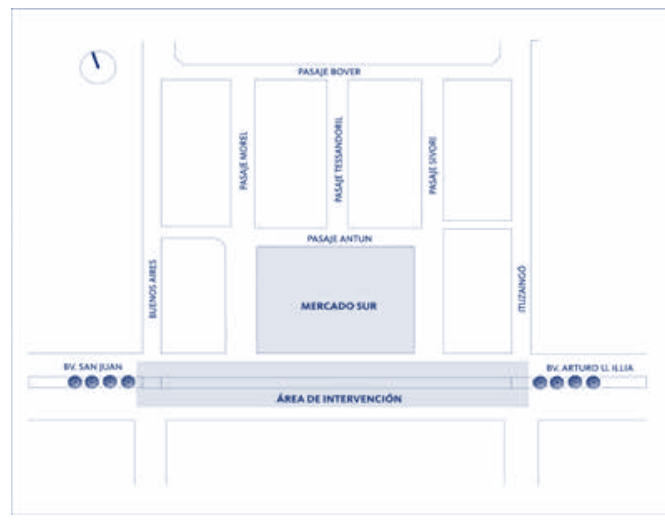

Imagen 01

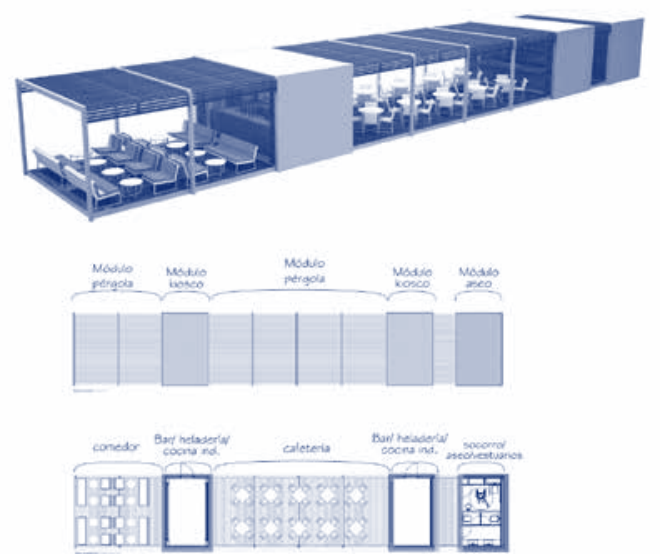

Imagen 02

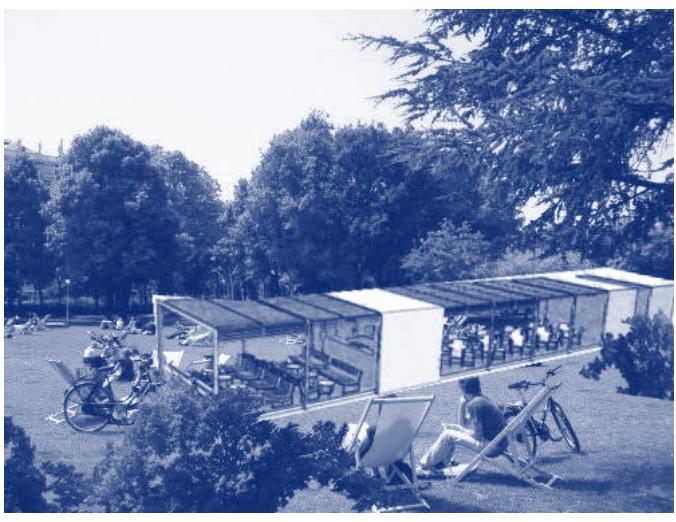

Imagen $0 \exists$

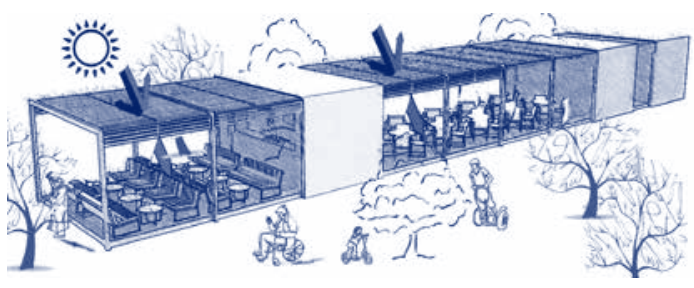

Imagen 04 


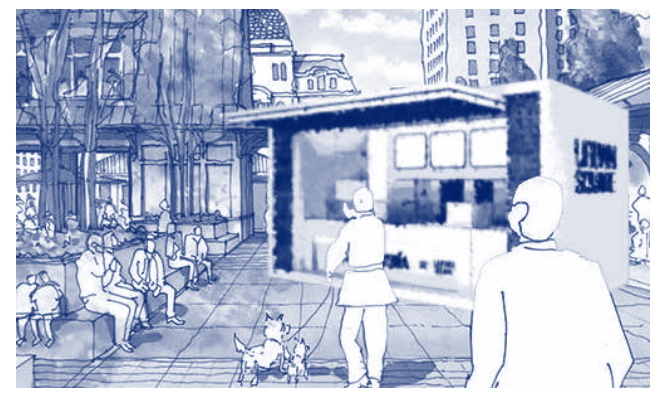

Imagen 05 : análisis propio.

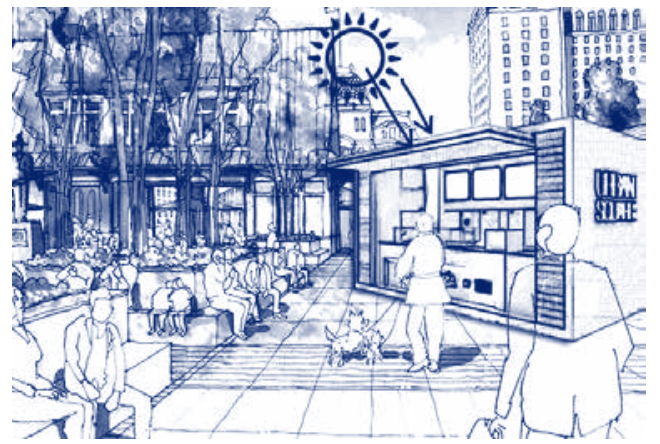

Imagen Ø6: análisis propio.

\section{Exploraciones}

Se realizaron diversas exploraciones en acero corten, contemplando las funciones propuestas para el sitio, trabajando el espacio intersticial con verde y articulando con las bicisendas. Trabajamos con los modelos All in Square, de In tenta

La propuesta sería con revestimiento exterior acero Corten perforado de $5 \mathrm{~mm}$ y con piso de acero antideslizante de $2 \mathrm{~mm}$. Algunos cerramientos se diseñaron en vidrio laminado, con soporte para gráfica.

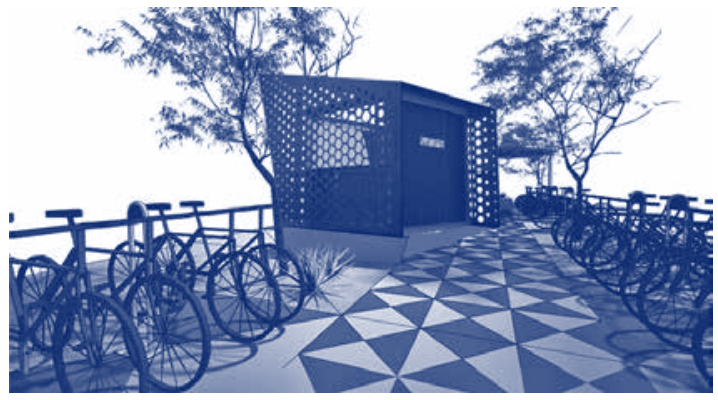

Imagen 07: propuesta exploratoria con bicicleteros.

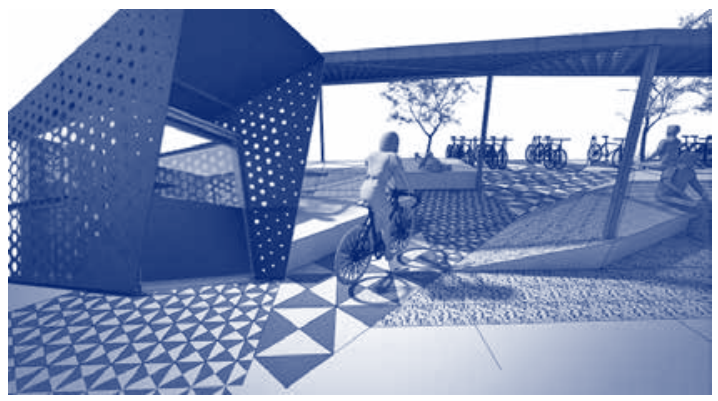

Imagen Ø8: propuesta exploratoria con estar al exterior.
Importancia del proyecto

Es un desafío para este grupo trabajar desde lo micro, equipamientos, a escala mayores y complejas como son los espacios de la ciudad. Se plantea con esta investigación el objetivo de regenerar sectores, de considerar actividades y proyectar para ellas con tecnologías de vanguardia, en una sinergia sostenible e inclusiva.

Mejorar el medioambiente, el espacio público, engrandecer con el diseño que conlleva orden y que contempla la función, la morfología y lo sustentable, mediante propuestas concretas de modelos de uso urbanístico y arquitectural y de equipamiento urbano. Se trata de integrar servicios para la vida urbana y para la naturaleza.

El diseño de espacios que ocupen los intersticios urbanos, que responda y se adecue a su entorno, o se destaque, para los usos que la sociedad demanda una tarea muy comprometida. Para ello es fundamental la comprensión del medio y una lectura clara detenida de su comportamiento. Este trabajo está aún en proceso.

\section{Agradecimientos}

Agradecemos la colaboración del equipo interdisciplinario, Ingeniero Hugo D Allegre, ingeniero Lucio Madussi, de la Facultad de Ingeniería, y al laboratorio de Robótica; a la Arq. Sara Boccolini, especialista en cálculo estructural, Arq. Raquel Landerberg, especialista en urbanismo; a la Arq. Eugenia Prigioni y al estudiante de sicología Pablo Martín Bobino.

\section{Referencias}

CEDOM (2007) Asociación Española de Domótica. Cuaderno de divulgación Domótica. 2 a ed. España, Barcelona: Aenor.

Constanza Cabezas (2014) "Claves Para Proyectar Espacios Públicos Confortables. Indicador del confort en el espacio público” 05 Jun 2014. ArchDaily. Recuperado 15 Sep 2014. <http://www.plataformaarquitectura. cl/cl/02-285882/claves-para-proyectar-espacios-publicos-confortables-indicador-del-confort-en-el-espacio-publico>

De Los Santos Aransay, A. (2009). Diseño de interacción centrada en el usuario, Doctorado, Universidad de Vigo, España

Diccionario Real Academia Española, Rae (actualizado 2012) [en línea], [consulta permanente], disponible en: http://www.rae.es.

Dominguez H. y Saez Vacas F. (2006) Domótica: Un enfoque sociotécnico, Madrid: Universidad Politécnica de Madrid.

Gonzalo G. E., et al. (2007). Diseño Bioclimático de Oficinas. Pautas para San Miguel de Tucumán. Centro de Estudios Energía y Medio Ambiente, Instituto de Acondicionamiento Ambiental. Tucumán: el autor.

Kcomt, Natalie Ché et al, (2010), An intelligent domotics system to automate user Actions, davy.preuveneers.be/publications/isami10b.pdf, recuperado en enero 2012.

Primer congreso de edificios inteligentes. (2013). España, 23 y 24 octubre. [Consulta octubre 2013]. Disponible en: http://www.congreso-edificiosinteligentes.es/

Romero Morales C. et al.(2006). Domótica e Inmótica: viviendas y edificios inteligentes. 2a ed. Madrid: Ra-Ma.

Schumacher, P. (2008) . Digital Cities. AD Architectural Design. Parametricism. A New Global Style for Architecture and Urban Design. 79 (4) 14-23

Urban Square, tipologías de microarquitectura, http://www.urbansquare.es/ seccionescatalogo.php?tp=4, consultado en marzo 2014 